\title{
La Banque mondiale, entre transformations et résilience
}

Article à paraître dans le numéro 53 de Critique Internationale, Novembre 2011 par Jean-Pierre Cling, Mireille Razafindrakoto et François Roubaud

Depuis sa création à Bretton Woods en 1944, la Banque mondiale a pris une importance de plus en plus grande, via la multiplication de ses organismes associés et l'extension du champ et du volume de ses actions. Conséquence de ce processus, son influence dans la définition et la mise en œuvre des politiques de développement dans les pays les plus pauvres est aujourd'hui sans commune mesure avec son importance somme toute assez modeste dans les flux internationaux de l'aide publique au développement (APD).

La profonde crise de légitimité engendrée par l'échec des politiques d'ajustement structurel à la fin des années 1990 a conduit l'institution à opérer un double aggiornamento. Dès le début des années 2000, la lutte contre la pauvreté a été placée au cœur des politiques de développement. Parallèlement, la Banque a mis l'accent sur l'importance de l'« économie politique » des réformes et sur la qualité de la gouvernance et des institutions dans le développement. Pourtant, en 2006, un dossier spécial de la revue L'économie politique mettait en doute le caractère effectif de ces réformes et titrait: «La Banque mondiale a-t-elle vraiment changé ? ». Sans nier l'importance des transformations mentionnées, nous tenterons de mettre en évidence leurs contradictions et poserons la question plus large de la possibilité de changements futurs.

Pour cela, nous soulignerons tout d'abord la difficulté qu'éprouve structurellement la Banque mondiale à se réformer. La première raison de cette difficulté est la contradiction « ontologique » entre les trois missions de la Banque mondiale dont la coexistence est dans la pratique source de nombreuses tensions. En tant qu'institution financière, la Banque mondiale doit se préoccuper de la rentabilité des financements qu'elle octroie ; en tant qu'agence de développement, elle doit chercher à lutter contre la pauvreté ou du moins à la réduire ; en tant que centre de recherche sur le développement, elle œuvre au service des deux fonctions précédentes (dont les intérêts ne sont pas forcément compatibles) et de la communauté du développement en général ${ }^{1}$. Contrainte de remplir simultanément ces trois missions, la Banque mondiale ne peut résoudre ces difficultés que de façon superficielle. La seconde

${ }^{1}$. Ces trois fonctions sont souvent résumées sous le terme de «banque d’affaires », de «banque de développement » et de «banque de savoirs ». 
raison est le rôle dominant des États-Unis et des grands pays industrialisés et leur refus de renoncer au mode de fonctionnement de l'institution, malgré la contestation croissante des pays en développement, plus particulièrement des pays émergents.

Nous montrerons ensuite l'enfermement de cette institution dans une vision étriquée du développement fondée sur le paradigme prégnant du marché, vision favorisée par l'hégémonie que la Banque mondiale a conquise au fil des années. Ainsi, l’adoption des stratégies de lutte contre la pauvreté ne remet pas fondamentalement en cause le «post-consensus de Washington » qui continue d’inspirer les politiques qu’elle préconise.

La crise financière internationale a fourni à la Banque mondiale une occasion inespérée de se remettre en selle. Ce nouvel impetus s'est notamment traduit par une montée massive des engagements en 2009-2010. Il semble néanmoins qu'au-delà de changements marginaux dans son mode de fonctionnement la "grande transformation » attendue n'ait pas eu lieu, ce manque venant renforcer le diagnostic pessimiste relatif à son incapacité à engager des réformes de l'intérieur.

En tant qu'économistes, nous aborderons la question du changement principalement du point de vue des politiques publiques, et ce dans une perspective d'économie politique. Notre analyse permettra néanmoins d’évoquer le changement institutionnel de la Banque mondiale sur le temps long, à la fois dans ce qu'elle fait et dans ce qu'elle est, selon les trois composantes identifiées dans l'introduction de ce dossier (réformes institutionnelles, reconfiguration de pouvoir et changement cognitif et normatif) ${ }^{2}$.

Nous montrerons qu'en dépit de changements profonds et continus, bien qu'à des degrés divers, sur ces trois fronts la Banque mondiale fait preuve d'une remarquable résilience : en s’emparant des nouvelles thématiques « à la mode ", elle réussit à maintenir son leadership international et à assurer le pouvoir normatif de ses théories du développement et de ses prescriptions, tout en les recyclant systématiquement dans une perspective étroite au service du marché.

2. Voir dans ce dossier l'introduction de Olivier Nay et Franck Petiteville, « Analyser le changement dans les organisations internationales ». 
La Banque mondiale, hegemon des politiques de développement

À l'origine, la Banque mondiale n'était qu'une petite organisation créée en marge des accords de Bretton Woods, dont le véritable enjeu - la gouvernance monétaire et financière internationale - s'est traduit par la création du Fonds monétaire international (FMI). La principale organisation de la Banque mondiale, la Banque internationale pour la reconstruction et le développement (BIRD), avait, comme son nom l'indique, pour fonction première la reconstruction de l'Europe après la seconde guerre mondiale. L'aide au développement ne s’est affirmée progressivement qu'après la reconstruction, avec l'essor des pays en développement et l'accès à l'indépendance des anciennes colonies.

\section{Une institution devenue protéiforme}

La Banque mondiale fonctionne comme une banque d'investissement finançant les pays en développement (PED). Durant les premières décennies de son existence, ces financements, destinés seulement aux États, avaient une structure sectorielle très concentrée : il s’agissait pour l'essentiel de grands projets d'infrastructures comme les constructions de routes et de barrages. Or il est apparu très vite que de nombreux pays pauvres n'avaient pas les moyens d'emprunter auprès de la BIRD qui prêtait au taux du marché. De plus, le fait que beaucoup de projets de développement (dans l'éducation et la santé, par exemple) aient une rentabilité économique incertaine créait des problèmes de remboursement des emprunts contractés. Pendant plusieurs années, la Banque mondiale a ainsi refusé d'intervenir dans l'agriculture et le social, malgré les besoins énormes de ces deux secteurs ${ }^{3}$.

La création en 1960 de l'Agence internationale pour le développement (AID) a donc fait sauter un premier verrou qui freinait l'essor des financements accordés par la Banque. À la différence de la BIRD, l'AID accorde des prêts concessionnels (c'est-à-dire à taux réduit) aux pays à bas revenus ${ }^{4}$. Ses ressources proviennent de levées de fonds régulières (appelées « reconstitutions ») auprès de ses membres. L'AID a pris une importance de plus en plus grande dans les financements accordés aux PED par la Banque mondiale et l'encours de ses

3. Devesh Kapur, John P. Lewis, Richard Webb, The World Bank: Its First Half Century, Washington, D.C., The Brookings Institution, 2 tomes, 1997 ; Michele Alacevich, The World Bank's Early Reflections on Development: A Development Institution or a Bank?, Policy Research Working Paper $\mathrm{n}^{\circ} 4670$, Washington, D.C., The World Bank, 2008, p. 1-21.

4. Les pays éligibles aux financements de l'AID sont ceux dont le niveau de revenus (PIB/habitant) est inférieur à environ 1000 dollars/habitant. 
prêts a même dépassé celui de la BIRD à partir du milieu des années $2000^{5}$. Ainsi la Banque mondiale a-t-elle progressivement élargi son champ d'intervention pour couvrir l'ensemble des services publics (éducation, santé, logement social, adduction d’eau, etc.).

Durant les premières années, les interventions de la Banque ont été limitées par ses statuts qui l'obligeaient à n'accorder que des prêts garantis par les Etats, autrement dit au secteur public. Très vite cependant, la Banque a souhaité élargir son champ au secteur privé. Passant outre la volonté de ses fondateurs, elle a créé en 1956 la Société financière internationale (SFI) qui fonctionne comme une banque d'affaires, se devant donc d'être rentable ; la croissance des financements accordés par la SFI au cours de ces dernières décennies et jusqu’à la crise a été supérieure à celle des financements tant de la BIRD que de l’AID.

\section{Une montée quasi ininterrompue des financements}

Depuis la création de la Banque mondiale jusqu'aux années 1980, le montant des financements annuels exprimés en dollars constants a doublé chaque décennie.

Cette expansion à un rythme exponentiel a été favorisée par la création des organismes associés, en particulier de l'AID. La multiplication progressive des secteurs d'intervention de la Banque est tout aussi remarquable. Interrompant le « tout-infrastructures », la présidence de Robert McNamara (1968-1981) a mis l'accent sur les prêts à l'agriculture et, dans une moindre mesure, sur les financements sociaux ${ }^{6}$.

Cette stratégie «attrape-tout » a été intensifiée sous la présidence de James Wolfensohn (1995-2005) et poursuivie ensuite, à tel point qu'aujourd'hui rares sont les secteurs qui ne font pas l'objet de financements de la Banque mondiale. Conformément à son mandat, l'institution s’est vue confier dans les années 2000 la coordination de la reconstruction des pays ayant subi des guerres ou des cataclysmes naturels (Irak, Afghanistan, Haïti). Lors du sommet de Pittsburgh en septembre 2009, le G20 lui a confié la création d'un Fonds pour la sécurité alimentaire mondiale. Plus généralement, la Banque a affirmé ses ambitions dans la gestion des biens publics mondiaux: un Fonds pour l'environnement mondial et des Fonds d'investissement climatiques dotés de budgets de plusieurs milliards de dollars ont été ainsi créés au cours des années 2000.

5. À la suite de la crise internationale, la remontée des prêts de la BIRD a permis à ces derniers de dépasser ceux de l’AID à la fin des années 2000.

6. Toutefois, contrairement à ce qui est souvent écrit, le rythme de croissance global des financements ne s'est pas accru sous R. McNamara, mais a seulement poursuivi sur sa lancée. Jean-Pierre Cling, François Roubaud, La Banque mondiale, Paris, La Découverte, 2008. 
À partir des années 1990, l'essor des financements de la Banque mondiale s'est interrompu, ceux-ci tendant même à décroître globalement en dollars constants dans les années 2000, avant la crise économique mondiale. Or celle-ci ayant généré une demande considérable de financements de la part des PED, les engagements financiers de la Banque mondiale ont connu une remontée spectaculaire, faisant plus que doubler en 2009-2010 par rapport à la moyenne des années 2000.

\section{L’acquisition progressive d'un leadership sur les politiques de développement}

L’objectif et le contenu des politiques menées par la Banque mondiale ont considérablement évolué au cours de ces dernières décennies. Les années 1980 ont vu le démarrage des programmes d'ajustement structurel (PAS) menés de concert avec le FMI, ce dernier se réservant la dimension budgétaire et macroéconomique des programmes et laissant à la Banque les politiques sectorielles et de libéralisation interne (privatisations).

Nous l'avons dit, la montée en puissance de la Banque mondiale en termes de financements accordés était régulière depuis sa création, mais c’est le lancement des PAS, au début des années 1980, qui a consacré le leadership de la Banque mondiale (et du FMI) sur les politiques de développement.

Les PAS sont nés des difficultés croissantes rencontrées par l'aide projet dans le contexte de la crise économique subie par les PED à la fin des années 1970 : en effet, l'impact conjoint des deux chocs pétroliers (1973 et 1978) et de la montée des taux d’intérêt américains a débouché sur la crise de la dette du début des années 1980. De nombreux PED étant contraints de s'adresser aux institutions de Bretton Woods pour des financements à la balance des paiements, la Banque mondiale et le FMI ont alors mis en place un nouveau mode d'intervention.

Le lancement des PAS a offert à la Banque mondiale l'occasion d'accroître son pouvoir en lui procurant « un siège à la table » des décideurs. La définition des conditionnalités associées au financement de ces programmes lui a conféré après plusieurs décennies de montée en puissance une influence déterminante sur les politiques économiques menées par les PED.

L'accroissement de cette influence, notable à partir des années 1980, résulte en fait d'une combinaison de l'adoption des nouvelles politiques d'ajustement structurel et de l'influence intellectuelle de la recherche sur les politiques de développement menée au sein de la Banque mondiale.

Certes, la Banque effectuait des recherches sur le développement depuis sa création - son premier directeur-adjoint de la recherche, Rosenstein Rodan, fut le promoteur du big push -, 
mais c’est le président R. McNamara qui, dès le début des années 1970, a donné à la recherche l'importance qu'elle a aujourd'hui, en créant une vice-présidence en charge de ces questions. Ensuite, ce sont les PAS qui ont donné à cette recherche, consacrée essentiellement à l'économie du développement (le responsable de la recherche est l'économiste en chef de la Banque mondiale) et très marginalement aux autres disciplines des sciences sociales, les moyens d'une expérimentation à grande échelle.

L’institution s'est mise alors à concevoir les politiques économiques des pays engagés dans des PAS, la conditionnalité des financements accordés les contraignant à mettre ces politiques en œuvre. À partir du début des années 1980, elle est ainsi devenue le leader mondial de la recherche en économie du développement, grâce à la taille de son département de la recherche, à la qualité des travaux qui y étaient menés et, en grande partie aussi, à l'influence de ces derniers sur les politiques des PED.

Les PAS se sont inscrits dans l'idéologie libérale résumée quelques années plus tard par John Williamson $^{7}$ sous le label de « consensus de Washington » (stabilisation macroéconomique ; libéralisation externe et interne). Toutefois, même si la prégnance de cette conception libérale dans le discours de la Banque est indéniable depuis plusieurs décennies, le paradigme de développement porté par l’organisation n’a cessé d'évoluer. Le contenu des rapports sur le développement dans le monde (World Development Report ou WDR) publiés chaque année par la Banque mondiale témoigne de cette évolution analysée en détail par Shahid Yusuf ${ }^{8}$ : ainsi, au cours de ces trente dernières années, la Banque a successivement mis l'accent sur la lutte contre la pauvreté (WDR 1980), au moment même où elle lançait les PAS, puis sur l'importance de l'intégration internationale, sous l'impulsion de son économiste en chef Anne Krueger (WDR 1987), sur le rôle de l’État (WDR 1997), réhabilité après presque deux décennies d'ajustement structurel, enfin, sur l'importance de l'agriculture (WDR 2008), oubliée pendant plus de deux décennies. Il faut cependant relativiser les effets de ces évolutions dans la mesure où il existe un certain décalage entre la façon dont la Banque présente le développement dans ses principales publications, tel le WDR, et la pratique sur le terrain lestée d'une grande inertie.

7. John Williamson (ed.), Latin American Adjustment: How Much Has Happened?, Washington, D.C., Institute for International Economics, 1990.

8. Shahid Yusuf (ed.), Development Economics through the Decades: A Critical Look at 30 Years of the World Development Report, Washington, D.C., The World Bank, 2009. 
Un changement de cap face à la crise de légitimité des années 1990

Plusieurs éléments ont conduit à l'apparition d’une crise de légitimité de la Banque et plus généralement des institutions de Bretton Woods durant les années 1990. Tout d’abord, l'échec flagrant des politiques d'ajustement structurel, compte tenu de leur impact en termes de montée de la pauvreté et de dégradation des indicateurs de développement humain dans les pays les plus affectés, en Afrique notamment. Ensuite, l'échec de l'initiative d'allègement de la dette des pays pauvres très endettés (PPTE) lancée en 1996 par les institutions de Bretton Woods. Enfin, le contexte général de «fatigue de l'aide». À cet égard, les critiques de William Easterly ${ }^{9}$ considérant l’APD comme largement inefficace, voire nocive, ont été d'autant plus cruelles qu'elles venaient d'un ancien de la Banque mondiale. Celle-ci s'est d'ailleurs vue prise sous les feux d'une double salve de critiques venues des deux bords de l'échiquier politique : de la droite conservatrice américaine, qui stigmatisait le gaspillage de ressources publiques au profit d'États autoritaires et corrompus (trade not aid) et qui, de façon ironique, intentait à la Banque le «procès en bureaucratie » qu'elle-même instruisait à l'encontre des $\mathrm{PED}^{10}$; de la gauche, sur fond de rejet de la libéralisation largement porté par les mouvements altermondialistes ${ }^{11}$.

Même si la Banque mondiale avait toujours fait preuve, du moins en apparence, d'une grande plasticité dans les politiques menées et dans son discours sur le développement, le lancement en 1999 des politiques de lutte contre la pauvreté a constitué une rupture indéniable, tant sur le fond que sur la forme. Face à une crise de légitimité qui menaçait de remettre en question son existence même, elle a été contrainte pour la première fois de se livrer à une autocritique publique et d'annoncer une profonde transformation de ses politiques et de son mode de fonctionnement avec les PED. Abandonnant formellement les politiques d'ajustement structurel, elle a alors proposé, de concert avec le FMI, de nouvelles stratégies de réduction de la pauvreté. Cette innovation, au demeurant lancée dans l'urgence et l'improvisation, compte

9. William R. Easterly, The Elusive Quest for Growth: Economists' Adventures and Misadventures in the Tropics, Cambridge MA, MIT Press, 2001.

10. Allan H. Meltzer, Report of the International Financial Institution Advisory Commission («Rapport Meltzer»), Congrès des États-Unis, Washington, D.C. (http://www.house.gov/jec/imf/meltzer.pdf), mars 2000 ; Larry Summers, Remarks by Larry Summers at the World Bank Country Directors Retreat », Speech to World Bank Country Directors, mai 2001. Cité dans Eurodad, Many Dollars, Any Change? Bruxelles, European Network on Debt and Development, octobre 2001.

11. Christian Chavagneux, "Lutte contre la pauvreté : les enjeux politiques d'un slogan », Politique africaine, 82, juin 2001, p. 161-168. 
tenu de la crise de légitimité évoquée, a été suivie en 2000 par l’adoption dans le cadre des Nations unies des Objectifs du Millénaire pour le Développement (OMD). Compte tenu de l'influence des institutions de Bretton Woods sur les politiques de développement, les nouvelles stratégies de la Banque mondiale ont été adoptées en même temps que les OMD par l’ensemble de la communauté internationale.

\section{De l'ajustement structurel à la lutte contre la pauvreté}

Conformément à ces nouvelles dispositions, chaque pays à bas revenus est appelé à définir sa stratégie nationale, qui est consignée dans un Document stratégique de réduction de la pauvreté (DSRP). Ces politiques se sont traduites par trois innovations majeures, dont chacune présente toutefois des limites ${ }^{12}$.

Première innovation, les nouvelles stratégies promues par la Banque mondiale ont permis de mettre la réduction de la pauvreté - et non l'ajustement structurel - au centre du développement. Alors que les premières tentatives dans ce sens de R. McNamara avaient été abandonnées rapidement après son départ (le WDR 1990 fixait déjà pour objectif la réduction de moitié de la pauvreté à l’horizon 1995), la Banque mondiale fixe désormais une finalité claire à son action, comme l'indique la devise inscrite dans le hall d'entrée de son siège à Washington : « Notre rêve : un monde sans pauvreté ».

Si l’objectif central des politiques est identique pour tous les PED (la réduction de la pauvreté dans ses différentes dimensions telles que définies par les OMD), le contenu des politiques mises en œuvre pour atteindre cet objectif diffère en principe selon les pays. Pour la première fois la Banque mondiale reconnaît que les PED présentent chacun des spécificités et qu’il n’existe pas un seul modèle de développement (rejet du « one size fits all »).

Cet objectif mobilisateur a contribué à la progression régulière des ressources obtenues par l’AID pour le financement des pays à bas revenus dans les années 2000. Ces ressources ont été canalisées dans le cadre de nouveaux instruments d'intervention mis au point par la Banque mondiale dans ces pays : les Crédits stratégiques de réduction de la pauvreté (Poverty Reduction Strategy Credits ou PRSC). Les pays en question ont également bénéficié d'une remontée plus générale de l'APD et de l'allègement de la dette finalement intervenu après une longue attente dans le cadre de l'Initiatitve PPTE renforcée et de l'Initiative pour l’Allègement de la dette multilatérale.

12. Jean-Pierre Cling, Mireille Razafindrakoto, François Roubaud (dir.), Les nouvelles stratégies internationales de lutte contre la pauvreté, Paris, Economica, 2003 (paru en anglais sous le titre, New International Poverty Reduction Strategies, Londres/New York, Routledge, 2003). 
Si une partie seulement de ces financements a transité par la Banque mondiale, celle-ci a toutefois joué un rôle catalyseur dans une nouvelle dynamique financière de l'APD. La sécurisation des dépenses sociales (santé et éducation) dans le cadre des allègements de la dette est également à mettre en grande partie à l'actif de la Banque mondiale, qui a elle-même accru la part de ce secteur dans ses financements.

Cependant, ces points positifs ne doivent pas conduire à passer sous silence les nombreuses faiblesses dans le contenu de ces nouvelles stratégies. Dans la pratique, les DSRP sont relativement uniformes et se composent pour l'essentiel d'un cadre macroéconomique destiné à favoriser la croissance, complété par des politiques sectorielles ciblées. Ils n’intègrent pas vraiment de politiques destinées à rendre la croissance plus favorable aux pauvres. La question sensible de la réduction des inégalités économiques et sociales, pourtant essentielle dans la lutte contre la pauvreté, n’est pratiquement jamais abordée. Globalement, les DSRP reprennent pour beaucoup les politiques antérieures, reconditionnées avec l'ajout de la lutte contre la pauvreté. Finalement, après avoir souligné la nécessité de prendre en compte les spécificités nationales, en vertu de son rôle attendu et de ses prérogatives à l'échelle internationale dans la préconisation de politiques de développement, il s’avère que la Banque mondiale ne se résout pas à abandonner l'idée qu'un même modèle puisse être appliqué à tous les PED.

Deuxième innovation, les DSRP reposent sur un principe d'appropriation des politiques par les pays concernés, qui sont censés les mettre en œuvre. L’objectif est de renoncer aux conditionnalités imposées, reconnues comme inopérantes, et d'établir une nouvelle relation plus égalitaire entre les acteurs du développement. Les PED - et non plus la Banque mondiale ou le FMI - sont censés concevoir eux-mêmes leurs stratégies de réduction de la pauvreté, dans le cadre d’un processus participatif organisé avec leur société civile.

Or ces stratégies reposent encore et toujours sur une forme de conditionnalité. En effet, leur adoption est la condition pour qu'un pays bénéficie de l'allègement de la dette et des financements de la Banque mondiale et du FMI. Dès lors, la tentation est forte pour les PED de définir des stratégies susceptibles de plaire à ces institutions, d'autant que jusqu'en 2006 les DSRP devaient être validés par un comité d'évaluation composé de représentants des deux institutions de Bretton Woods.

Enfin, troisième innovation, la nécessité d’assurer un véritable suivi-évaluation des politiques de développement est affirmée, chaque pays devant notamment situer ses progrès dans l'atteinte des OMD à l'horizon 2015. La Banque mondiale, quant à elle, s'est vue confier la responsabilité du suivi de ces OMD et publie à ce titre un rapport annuel. Ce rôle est 
largement dû à la ténacité de ses efforts en matière de collecte de données d'enquêtes, en particulier sur les conditions de vie des ménages et sur la pauvreté, dans le cadre du Living Standard Measurement Survey. Ces enquêtes lui permettent en effet de produire des connaissances de première main sur la configuration des sociétés en développement, et donc d'alimenter le débat public, tout en s'adjugeant le monopole de la production des chiffres officiels de la pauvreté dans le monde. Le fait de fixer des objectifs quantifiés permettant d'évaluer l'impact des politiques de développement est incontestablement un progrès, comme le sont les politiques elles-mêmes, tant dans leur contenu que dans leur mise en œuvre. Cela dit, il était notoire, bien avant la crise de 2008, que les OMD ne seraient pas atteints dans la plupart des pays concernés. Quant à l'évaluation opérée par la Banque mondiale, elle demeure dans la pratique (au-delà de la vision globale des OMD) extrêmement sommaire, faute de données et de méthodes adéquates.

\section{La prise en compte de l'économie politique}

Jusqu’aux années 2000, la Banque mondiale s’est limitée à une approche « économiciste » du développement. Cette approche traditionnelle, restrictive, était justifiée officiellement par ses statuts, qui lui interdisent d’intervenir dans le champ politique (article IV, section 10).

Comme le rappelle Jean Coussy ${ }^{13}$, une première brèche a été ouverte en 1993 avec la publication de l'étude sur les pays émergents asiatiques, The East Asian Miracle ${ }^{14}$. Ce rapport préparé à l'initiative du Japon contredisait la thèse dominante selon laquelle le succès de ces pays était dû avant tout à leurs politiques de libéralisation commerciale et de croissance par l'exportation. Prenant le contre-pied de cette thèse, il mettait en avant l'impact crucial des interventions de l'État et des politiques publiques (protection commerciale, subventions, etc.) dans l'émergence de ces pays.

Dans sa conception la plus étroite, la prise en compte du « politique » par la Banque mondiale date en fait des premiers travaux publiés dans les années 1990 sur la sélectivité de l’aide et le rôle de la « bonne gouvernance ». En 1998, les auteurs d'une étude célèbre intitulée Assessing Aid ${ }^{15}$ affirmaient ainsi que 10 milliards d’aide dirigés vers des pays bien gouvernés pouvaient

13. Jean Coussy, «L’économie politique à la Banque mondiale : la fin des exclusives ? » L'économie politique, 30, 2006, p. 7-20.

14. Banque mondiale, The East Asian Miracle: Economic Growth and Public Policy, A World Bank Policy Research Report, Oxford/New York, Oxford University Press, 1993.

15. Banque mondiale, Assessing Aid. What Works, What Doesn't, and Why, A World Bank Policy Research Report, Oxford/New York, Oxford University Press, 1998. 
faire sortir 25 millions de personnes de la pauvreté, contre seulement 7 millions dans le cas d'une aide non ciblée.

Indicateur de l'influence de la Banque mondiale, le principe de sélectivité de l'aide a été adopté formellement par les pays donateurs dans le cadre du Consensus de Monterrey en 2002. La validité empirique de ce concept repose sur des bases extrêmement fragiles comme l'a reconnu la Banque mondiale elle-même par la suite ${ }^{16}$. On peut par ailleurs s'interroger, d'un point de vue moral, sur une sur le bien-fondé d'une politique consistant à punir les populations opprimées des pays pauvres en réduisant (voire en supprimant) l'aide dont elles ont pourtant cruellement besoin. Le principe de sélectivité présente cependant deux avantages pour l'institution et pour les bailleurs de fonds d'une manière générale: d'une part, il participe de l'entreprise de «re-légitimation » de l'aide en prétendant avoir inventé une recette miracle qui semble relever du bon sens; puisqu'on sait maintenant verser l'APD efficacement, on peut accroître les budgets ; dans la pratique, cette innovation a effectivement contribué à la remontée de l'APD; d'autre part, ce concept revient à faire porter la responsabilité de l'échec des réformes sur les PED eux-mêmes et non plus seulement sur les donateurs, en l'occurrence la Banque mondiale ; dès lors, si les politiques jadis promues par cette dernière (l'ajustement structurel en particulier) n’ont pas marché, c’est parce que ces pays étaient mal gouvernés !

Au cours des années suivantes, la Banque mondiale a lourdement investi dans la mise au point de divers indicateurs destinés à mesurer le degré de «bonne gouvernance » dans le monde : World Governance Indicators (WGI) ; Country Policy and Institutionnal Assessment (CPIA), etc. Certes, ces indicateurs fournissent des informations utiles sur la qualité des institutions dans les PED, mais ils souffrent de biais manifestes ${ }^{17}$. En effet, les États-Unis étant systématiquement les mieux classés, il ressort de façon à peine implicite que la cause du sousdéveloppement est la mauvaise gouvernance et que, pour se rapprocher du niveau de développement des États-Unis, il convient d’adopter leurs institutions. Or, en dépit des imperfections de ces indicateurs, l'un d'eux, le CPIA, est directement utilisé pour déterminer l'allocation géographique des fonds de l'AID.

Dans le domaine de la prise en compte de l'économie politique et des institutions, le tournant majeur qu'a été le lancement des nouvelles stratégies de réduction de la pauvreté et la

16. Abhijit Banerjee, Augus Deaton, Nora Lustig, Kenneth Rogoff, An Evaluation of World Bank Research, 1998-2005, Washington, D.C., Banque mondiale, 2006.

17. M. Razafindrakoto, F. Roubaud, "Are International Databases on Corruption Reliable? A Comparison of Expert Opinions Surveys and Household Surveys in sub-Saharan Africa », World development, 38 (8), août 2010, p. 1057-1069. 
publication du Rapport sur le développement dans le monde (2000) s’est confirmé au cours des années suivantes. On peut voir dans cette évolution l’influence de Joseph E. Stiglitz, alors économiste en chef de la Banque mondiale (1997-2000), mais surtout la reconnaissance des conséquences des erreurs passées.

Les nouvelles politiques participent tout d'abord d'une entreprise de réhabilitation de l'État, tant décrié pendant les décennies d’ajustement structurel. Dans sa démarche de mea culpa, la Banque mondiale est allée jusqu’à reconnaître que « les réformes [s’étaient] concentrées sur les distorsions induites par les interventions de l’État alors que les contraintes mordantes venaient des imperfections de marché ${ }^{18}$. En d'autres termes, dans les pays à bas revenus, l’État est en charge de la définition et de la mise en œuvre des stratégies de réduction de la pauvreté, mais sans avoir la capacité et les ressources suffisantes pour mener à bien une telle tâche.

Avec les nouveaux concepts d'appropriation des politiques (ownership) dans le cadre des processus participatifs, d'insertion des pauvres (empowerment) et de responsabilité démocratique des institutions publiques (accountability), la Banque mondiale est entrée de plain-pied dans le champ politique auparavant délaissé. En un temps très court, elle s'est approprié deux théories situées en marge de la vulgate de l'économie standard, mais toutes deux couronnées par le prix Nobel d’Économie : l'économie du développement humain promue déjà depuis plusieurs années par le PNUD (Amartya Sen et le concept de capabilities 1998), l'économie institutionnelle (Douglass North, 1993). De plus, alors qu'elle se l'était interdit auparavant, en vertu du principe de «non-incursion dans la sphère politique », elle s'est permis de critiquer la corruption endémique dans de nombreux PED (thème tabou jusque-là). En 2007, elle a ainsi créé, en coordination avec les Nations unies, le Stolen Asset Recovery (StAR), programme destiné à récupérer l'argent détourné par les dirigeants de ces pays.

Cependant, cet élargissement du champ de ses thématiques et de ses politiques s'est toujours fait implicitement au nom d'un objectif unique : l'efficacité du dieu-marché. Les institutions doivent être remodelées, voire construites de toutes pièces pour servir ce dernier (Building Institutions for Markets, WDR 2002) ${ }^{19}$, les populations marginales doivent s'y intégrer pleinement, grâce à l'amélioration des opportunités pour les pauvres qui demeure le premier

18. «Reforms Focused on Government Failures, When the Binding Constraints Were Market Failures », dans World Bank, Economic Growth in the 1990s: Learning from a Decade of Reform, Washington, D.C., The World Bank Policy Research, 2005.

19. World Bank, World Development Report 2002. Building Institutions for Markets, New York, Oxford University Press, 2001. 
objectif (Attacking Poverty, WDR 2000/01) ${ }^{20}$. Cette vision réductrice conduit la Banque mondiale à détourner les théories et concepts dont elle s'empare, voire à les vider de leur substance, comme le montre Anne-Emmanuèle Calvès ${ }^{21}$, au sujet de «l'empowerment ». Initialement conçu par les chercheurs et activistes (féministes en particulier) comme un processus de transformation des relations de pouvoir, donc collectives et politiques, entre individus et groupes sociaux, l'empowerment est devenu entre les mains de la Banque mondiale individualiste, dépolitisé, vertical, instrumental et réduit à sa seule dimension économique d'extension des relations marchandes.

La même critique peut être faite à propos de la gouvernance. Comme le souligne Mushtaq $\mathrm{Khan}^{22}$, cette dernière est appréhendée dans une vision étroite, dont la fonction est de corriger les imperfections de marché (market-enhancing governance capabilities), alors même que ce type de réformes ne peut ni être mené à bien faute de ressources ni promouvoir une croissance durable. La gouvernance démocratique se voit marginalisée au profit d'une approche quasi comptable de la gouvernance d'entreprise, budgétaire ou judiciaire. Les exemples analogues sont nombreux, de même que ceux relatifs aux institutions, la Banque engageant les PED à adopter les formes institutionnelles des pays développés, principalement d'inspiration anglosaxonne, aux dépens d'arrangements plus complexes et endogènes ${ }^{23}$. Ce fétichisme de la forme s'accompagne d'un biais évident, qualifié de «property rights reductionism » par Dani Rodrik $^{24}$, en faveur des droits de propriété privée censés être en tout temps et en tout lieu plus efficaces que les autres.

D’ailleurs, la dernière phrase du tout récent discours du Président Robert Zoellick évoquant, entre autres, le «printemps arabe » suscite le doute ${ }^{25}$ : «Cette année [2011] (...) sera-t-elle (...) l'année où l'on a découvert que la participation citoyenne compte pour le développement? ». Cette déclaration n’amène-t-elle pas à s'interroger sur l'importance

20. World Bank, World Development Report. 2000/01. Attacking Poverty, New York, Oxford University Press, 2000.

21. Anne-Emmanuèle Calvès, " "Empowerment": généalogie d'un concept clé du discours contemporain sur le développement », Revue Tiers Monde, 200, octobre-décembre 2009, p. 735-749.

22. Mushtaq H. Khan, "Governance and Development: The Perspective of Growth-enhancing Governance ", dans GRIPS Development Forum (ed.), Diversity and Complementarity in Development Aid: East Asian Lessons for African Growth, Tokyo, National Graduate Institute for Policy Studies, 2008.

23. Ha-Joon Chang, «Understanding the Relationship between Institutions and Economic Development: Some Key Theoretical Issues », dans Ha-Joon Chang (ed.) Institutional Change and Economic Development, New York, United Nations University Press, 2007, p. 17-34.

24. Dani Rodrik, "Getting Institutions Right », mimeo, Kennedy School of Government, Harvard University, 2004.

25. Robert B. Zoellick, «Moyen Orient et Afrique du Nord : Un nouveau contrat social pour le développement », discours prononcé au Perterson Institute for International Economics, 6 avril 2011. 
réellement accordée aux fameux «processus participatifs », pourtant censés être au cœur des DSRP depuis une décennie ? Bien que l'entrée affichée en politique ne puisse se résumer à une simple entreprise de re-légitimation discursive, tant elle remodèle l'action de la Banque, elle reste empreinte de contradictions à ce jour insurmontables.

Il semble finalement que la Banque mondiale ne fasse pas toujours ce qu'elle dit. Certes, elle se place à la pointe des théories à la mode, mais elle les reconditionne selon sa propre doxa (market oriented), et quand elle les met en pratique, elle introduit une nouvelle forme de conditionnalité, liée notamment à la corruption et à la gouvernance en général, pratique censée avoir été abandonnée avec l’ajustement structurel.

Des contradictions insurmontables?

Malgré les évolutions réelles de ces dernières années, la Banque mondiale demeure « engluée » dans des contradictions qui bloquent toute possibilité de transformation en profondeur. Ces contradictions sont liées à la fois au caractère tripartite de sa mission (financement, développement et savoirs) et à son mode de gouvernance, essentiellement dominé par les États-Unis.

\section{Une fonction financière toujours dominante}

Depuis la création de l'institution, la plupart de ses présidents - tous américains - sont venus du monde de la finance, mais aucun du monde du développement. La «culture du décaissement » dénoncée il y a déjà vingt ans par le rapport Wapenhans (1992) n’a pas disparu, ni même régressé. Les opérationnels de l'institution sont d'ailleurs incités à dépenser, d'autant que le système de rotation des postes leur permettra de ne plus être en place au moment de la réalisation du projet, et donc d'être déchargés de toute responsabilité. La contradiction est indépassable entre l’objectif de décaissement et de rentabilité des projets, qui encourage à financer des pays plus développés et mieux gérés, et l’objectif de développement, qui concerne plutôt les pays d'Afrique sub-saharienne. On en revient sous une formulation un peu différente au problème de la sélectivité de l'aide. Cette culture «bancaire » et ses conséquences sont encore plus marquées à la SFI pour laquelle l’objectif de développement est marginal. Un exemple extrême de cette contradiction est le cas de la Zambie, encouragée à s’endetter massivement dans les années 1970 dans le cadre d’une politique de grands projets 
qui l'a amenée au surendettement quelques années plus tard. Il est ironique que ce pays soit aujourd'hui présenté comme l'exemple qui justifie la sélectivité de l'APD par l’institution qui est largement responsable de ces errements.

La culture du décaissement va également à l'encontre des impératifs de «bonne gouvernance » et de responsabilité sociale et environnementale, dont le respect trop scrupuleux ne pourrait que ralentir le nombre et le montant des projets. Ces derniers sont censés émaner des pays bénéficiaires (demand driven), qui ont une prédilection pour les gros projets. Les fonctionnaires de la Banque sont donc confrontés à des demandes contradictoires, qui ne peuvent être résolues que dans le cadre d'un système « d'hypocrisie organisée » ${ }^{26}$. La mise en place d'un système d'évaluation totalement indépendante des financements de la Banque mondiale (ce qui n’est pas le cas aujourd'hui) pourrait contribuer, sans les résoudre totalement, à atténuer ces contradictions.

$\mathrm{Au}$ total, rien n'illustre mieux le caractère central de l'objectif de rentabilité de la Banque mondiale que le fait que le solde des transferts financiers entre la Banque mondiale et les PED (différence entre apports financiers aux PED et remboursements de ces pays) soit structurellement négatif depuis les années 1990, même si la crise internationale a provoqué une inversion de tendance (le solde devenant positif en raison de l'essor des financements). En effet, pour que la BIRD dégage un profit qui lui permette de fonctionner, le transfert financier net doit être structurellement négatif (sauf si les financements sont en croissance régulière ce qui n'était pas le cas avant la crise), c'est à dire que les remboursements d'emprunts doivent être supérieurs aux nouveaux engagements.

\section{Une activité de recherche sous influence}

Le fait de mener une mission de recherche dans une banque de développement est en partie un atout. Cette recherche est alimentée et enrichie par les connaissances de terrain de l'institution. Il arrive d'ailleurs que durant leur carrière les chercheurs pratiquent eux-mêmes des allers et retours entre travail opérationnel et travail de recherche. Sa présence physique et son pouvoir dans de nombreux PED fournissent en outre à la Banque mondiale un accès privilégié à l'information sur ces pays. Ainsi la recherche est-elle en prise sur les politiques de développement à la définition et à l'évaluation desquelles elle peut contribuer.

Pourtant, cette situation est également source de problèmes. D’abord, parce que la forte influence au niveau international des travaux de recherche menés en interne a pour

26. Catherine E. Weaver, Hypocrisy Trap: The World Bank and the Poverty of Reform, Princeton, Princeton University Press, 2008. 
contrepartie un contrôle étroit de cette production et des publications ${ }^{27}$. Ensuite, parce que l'une des fonctions essentielles de ces travaux consiste à valider et à promouvoir les politiques menées par la Banque mondiale. Les chercheurs promus sont donc ceux qui défendent ces politiques (David Dollar) tandis que les voix discordantes sont marginalisées, voire conduites à démissionner (J. Stiglitz, W. Easterly, par exemple) ${ }^{28}$.

La recherche permanente d'élèves modèles de la Banque mondiale, à grand renfort de publications et de chiffres, est un exemple de ce mode de fonctionnement. Le dernier en date de la liste est le Vietnam, considéré de façon étonnante comme un succès des politiques de libéralisation économique, alors même que ce pays a organisé un modèle propre d'économie mixte (État-marché) dans le cadre d'un système de parti unique dirigé par le Parti communiste $^{29}$.

En dépit d’une certaine pluralité de vues, l'ouverture de la Banque est donc relative, et in fine les décisions y sont bureaucratiquement (et idéologiquement) conditionnées. Certes, diverses sensibilités s’y expriment, mais toujours dans le cadre du prisme de l'économie dominante, comme le montre clairement la composition des chercheurs du département de la recherche et les documents de travail qu'ils publient. Dans ces conditions, la fonction de recherche au service des politiques présente un double visage : si la Banque est capable de s'approprier de nouveaux champs de recherche et de les porter au-devant de la scène (gouvernance, qualité et rôle des institutions, etc. ${ }^{30}$ ), ces thématiques sont systématiquement reconfigurées et reformatées dans le moule réductionniste des idées et des prescriptions qui pourraient en être tirées « pour faire fonctionner le marché », objectif apparemment indépassable.

Cette ouverture contrôlée de la Banque est confrontée à une triple difficulté interne : tout d'abord, les nouvelles idées sont loin d'être systématiquement appliquées sur le terrain (ceux qui parlent - dirigeants, chercheurs - ne sont pas ceux qui agissent), et rien ne prouve que des

27. Ce travers apparaît d'autant plus problématique que la Banque constitue (et revendique le fait d'être) le premier centre de recherche mondial en (économie du) développement, ce statut lui conférant un pouvoir normatif disproportionné, que J. Stiglitz dénonçait ironiquement comme devant être attaqué pour violation de la loi anti-trust. Joseph Stiglitz, "Remarks on Bank Research Evaluation », 2007 (http://siteresources.worldbank.org/DEC/Resources/84797-1109362238001/7264541164121166494/JES-bankResearchReviewanjesfin.pdf).

28. Voir J.-P. Cling, F. Roubaud, La Banque mondiale, op. cit..

29. J.-P. Cling, M. Razafindrakoto, F. Roubaud, La Banque mondiale à la recherche de modèles de développement : le cas du Vietnam, Hanoi, Éditions Tri Thuc, 2009.

30. Le rapport sur le développement dans le monde 2011, Conflit, Sécurité et Développement, paru au moment de la rédaction de cet article, est la plus récente illustration de cette capacité à s'approprier de nouvelles thématiques (ici la légitimité des institutions et le contrôle de la violence) et à en restreindre le champ (sécurité des citoyens, systèmes judiciaires et création d'emplois). 
changements significatifs aient été opérés à l'échelle des projets ${ }^{31}$; ce passage est d'autant plus difficile qu'aux échelons hiérarchiques intermédiaires et supérieurs, nombreux sont ceux qui revendiquent encore une vision étroite du Consensus de Washington; ensuite, les économistes qui constituent le gros des troupes de la Banque ne sont probablement pas les mieux placés pour formuler et conduire les réformes institutionnelles préconisées ; enfin, il existe un hiatus entre l'entrée de la Banque « en économie politique » et son absence de mandat politique ; certes, elle intervenait dans le champ politique auparavant, mais la prise en compte explicite des aspects sociopolitiques du développement rend sa position officielle « d'apolitisme » impossible à tenir ; d'ailleurs, la question du bien-fondé de l'ouverture au champ politique reste ouverte, si l'on s'en tient à l'usage qui en a été fait au nom de la démocratie lors de l'intervention militaire américaine en Irak, la Banque ayant été mobilisée massivement pour en gérer les conséquences.

Les rapports officiels d'évaluation de la recherche ont évoqué plusieurs de ces difficultés ${ }^{32}$, mais il nous semble peu probable, compte tenu des enjeux, que des mesures soient prises pour corriger ces pratiques. Le dernier rapport stratégique paru en 2010 s’intéresse ainsi avant tout aux thématiques de recherche à privilégier, non au mode d'organisation et de fonctionnement de cette recherche ${ }^{33}$. La recherche sur le développement y est toujours synonyme d'économie du développement, les apports des autres sciences sociales devant être pris en compte, mais sans que les recherches dans ces autres disciplines soient menées au sein de la Banque mondiale.

\section{Une gouvernance interne qui n'évolue qu’à la marge}

La Banque mondiale est contrôlée largement par les États-Unis qui nomment son président et exercent ensuite un contrôle direct de sa politique ${ }^{34}$. Ce pays est également le premier actionnaire de la Banque et dispose d'un droit de veto sur toutes les décisions importantes. Enfin, le fait que le siège de l'institution soit situé à Washington, à proximité de la Maison Blanche et du Trésor américain, renforce encore ce contrôle, d'autant que la diversité des

31. D. Rodrik, "Goodbye Washington Consensus, Hello Washington Confusion? A Review of the World Bank's Economic Growth in the 1990s: Learning from a Decade of Reform », Journal of Economic Litterature, XLIV, décembre 2006, p. 973-987.

32. A. Banerjee, A. Deaton, N. Lustig, K. Rogoff, An Evaluation of World Bank Research, 1998-2005, op. cit..

33. Development Economics Senior Vice Presidency, Research for Development, A World Bank Perspective on Future Directions for Research, Policy Research Working Paper n5437, Washington, D.C., The World Bank, 2010.

34. Catherine Gwin, «US Relations with the World Bank 1945-1992 », dans D. Kapur, J. P. Lewis, R. Webb, The World Bank: Its First Half Century, op. cit.. 
nations d'origine du personnel ne doit pas faire oublier que la plupart de ses cadres sont des diplômés d'universités américaines et fonctionnent donc selon un même moule théorique et idéologique avec l’anglais pour seule langue de travail.

Les grandes orientations politiques de la Banque mondiale sont directement influencées par les États-Unis. Citons, à titre d'exemple, la création de l'AID, proposée par un politicien américain et destinée à faire pièce à un projet concurrent défendu par l'ONU ; le tournant libéral du début des années 1980 consécutif à l'élection de Ronald Reagan et qui s’est traduit par le lancement des politiques d'ajustement structurel et une profonde réorganisation du personnel accompagnée de nombreux départs ; l’allocation géographique des financements, qui, dès le début, a répondu dans ses grandes orientations aux intérêts des États-Unis. Ces derniers ont même interdit à la Banque de financer leurs ennemis politiques : le Chili sous Allende (les financements n’ont repris après le coup d’État du général Pinochet) ; le Vietnam de 1973 à 1993, et jusqu’à la fin de l'embargo américain imposé à ce pays. Plus récemment, la Banque mondiale a été nommée responsable de la coordination de la reconstruction en Afghanistan et en Irak, après les guerres menées par les États-Unis dans ces deux pays.

Aucune remise en question de cette domination ne se profile à l'horizon, même si les ÉtatsUnis ont dû accepter sous la pression des grands pays émergents que le prochain président soit nommé dans le cadre d'un processus ouvert et concurrentiel. Or les conditions de désignation du nouveau directeur général du FMI, après la démission de Dominique Strauss-Kahn en 2011 (alors qu'il avait été annoncé que le poste serait ouvert à un candidat originaire d'un PED), augurent mal d'un changement du processus de désignation du président de la Banque mondiale. De surcroît, un abandon du droit de veto américain n’est toujours pas à l'ordre du jour, alors qu'il s'agirait d'un signal fort d'égalité entre les actionnaires de l'institution.

Contrepartie de cette domination persistante des États-Unis, et des pays européens dans une moindre mesure, les PED ont toujours une influence marginale au sein de la Banque mondiale ${ }^{35}$. La réforme de 2010 a peu changé la répartition des droits de vote et les PED restent minoritaires, même si la Chine (devenue le troisième actionnaire derrière les ÉtatsUnis et le Japon) et quelques pays émergents ont acquis davantage de poids. Le changement décidé à la Banque mondiale est ainsi moins ambitieux que celui décidé au FMI à l'automne 2010.

Globalement, il n’existe pas de justification à cette mainmise des pays développés sur le capital de la BIRD (le cas de l'AID financée par les contributions des pays développés est un

35. À cet égard, la nomination en 2009 d'un économiste en chef chinois (Justin Li) est évidemment à saluer, même si elle ne change pas fondamentalement le diagnostic. 
peu différent): puisqu'elle fonctionne sans soutien financier de ces pays, un contrôle majoritaire par les PED, qui sont ses principaux clients, serait plus justifié. Un tel changement pourrait d'ailleurs favoriser un système de feedback de la part des pays d'intervention de la Banque $^{36}$ : en appliquant le principe d'accountability à cette institution, celle-ci se sentirait responsable de l'impact des mesures qu'elle impose à ces pays (selon le même principe préconisé pour rendre les autorités nationales responsables devant leurs citoyens).

Enfin, en l'absence de véritable réforme de la gouvernance économique mondiale malgré la crise, la place de la Banque mondiale au sein du système international et ses missions demeurent inchangées. On peut regretter que la crise ne soit pas l'occasion d'une réforme importante de cette gouvernance mettant fin au poids déterminant du triumvirat constitué par la Banque mondiale, le FMI et l'OMC. Une meilleure articulation entre les institutions de Bretton Woods, qui aurait en particulier pour avantage de réduire la concurrence entre elles, est nécessaire, ainsi qu'une coordination accrue avec le système des Nations unies. Le «pêché originel » de la Banque mondiale et du FMI, nés sous l'égide de l'ONU mais qui n’ont eu de cesse ensuite de s'en affranchir, rend en effet bancale depuis l'origine la gouvernance économique mondiale. Seule une réforme globale, passant nécessairement par une redéfinition du poids des grandes puissances dans ces organisations, pourrait lever les contradictions majeures qui pèsent sur la Banque mondiale et sur les autres institutions internationales.

L’histoire de la Banque mondiale depuis sa création est faite d'un essor régulier accompagné de revirements stratégiques multiples. À chaque fois, la Banque mondiale est parvenue à surmonter ses difficultés et ses remises en cause et à rebondir en utilisant ses faiblesses pour devenir plus forte. Assénant tous azimuts son slogan It’s a learning process (« nous sommes dans un processus d'apprentissage »), elle a fait preuve d'une fausse humilité qui lui a permis de justifier toutes ses erreurs.

Les changements de politiques permanents de ces dernières décennies ont démontré une plasticité apparente indéniable. Le dernier en date a été celui de la fin des années 1990. Avec

36. W. R. Easterly, The White Man's Burden: Why the West's Efforts to Aid the Rest Have Done So Much Hill and So Little Good, New York, Penguin Press, 2006. 
la lutte contre la pauvreté comme nouvelle stratégie, la Banque mondiale a réalisé un coup de maître : non seulement elle a restauré sa légitimité gravement atteinte, mais en outre elle a obtenu un accroissement de ses ressources et renforcé son hégémonie par rapport aux autres donateurs sur le terrain.

Le changement de la Banque mondiale depuis ses origines ne fait aucun doute. Ses fondateurs et ses premiers agents (72 fonctionnaires contre plus de 10000 aujourd'hui) seraient probablement bien en peine de reconnaître l'institution dans sa configuration actuelle. Sous la pression combinée de facteurs internes et externes, la Banque mondiale a mis en œuvre les trois idéaux types de transformation affectant les organisations internationales (réformes institutionnelles, reconfiguration de pouvoir et changement cognitif et normatif).

En témoignent la création successive de ses différentes agences (AID, SFI, etc.), son leadership sur les grand fonds mondiaux nouvellement créés (environnement, sida, sécurité alimentaire, etc.) ainsi que le passage de l'ajustement structurel à la lutte contre la pauvreté et la prise en compte des aspects environnementaux, sociaux et de gouvernance, pour ne citer que les exemples récents les plus emblématiques. La Banque n'est pas seulement un diffuseur de normes, elle est aussi en quelque sorte un consommateur de normes: par exemple lorsqu'elle reprend à son compte les principes du développement humain du PNUD et les adopte pour sa conversion à la lutte contre la pauvreté ; ou, dans le domaine du développement durable, lorsqu'elle remodèle en profondeur son «identité environnementale ${ }^{37}$ sous l'influence des réseaux d'ONG du Sud et du Nord (transnational advocacy networks). Certes, les changements ont été plus timides dans le domaine des reconfigurations de pouvoir (voir la réforme marginale des droits de vote), mais la croissance du nombre d'États membres, les grandes transformations macro-historiques (décolonisation, fin de la guerre froide, phénomène d'émergence et de mondialisation) ont conduit à une atténuation de la mainmise exclusive du Nord en faveur d'un rôle croissant des pays du Sud (essentiellement les grands émergents). Ce déplacement progressif a été servi par le processus de décentralisation interne des structures de décision, initié dans le cadre du Strategic Compact sous la présidence de J. Wolfensohn, afin de mieux servir les demandes des pays bénéficiaires ${ }^{38}$.

37. Susan Park, «Norm Diffusion within International Organizations: A Case Study of the World Bank », Journal of International Relations and Development, 8, 2005, p. 111-141.

38. Daniel L. Nielson, Michael J. Tierney, Catherine E. Weaver, «Bridging the RationalistConstructivist Divide: Re-engineering the Culture of the World Bank », Journal of International Relations and Development, 9, 2006, p. 107-139. 
Pourtant, parallèlement, la Banque a fait preuve de sa capacité à préserver la fonction-objectif qu'elle cherche à tout prix à pérenniser, à savoir le maintien de son leadership mondial en matière de développement (politiques et recherche). Pour ce faire, elle a réussi à reprendre à son compte les débats scientifiques en vigueur, en réorientant les nouveaux concepts au service du même paradigme : l'extension permanente de la sphère du marché. Cette résilience s'explique par l'existence de deux facteurs qui se renforcent mutuellement et contrastent fortement avec ce que l'on peut observer à l'ONU en particulier. Le premier facteur est la concentration du pouvoir, sous la tutelle des États-Unis, entre les mains des grands pays développés, qui résistent à toute modification fondamentale de leurs prérogatives. Le second est la profonde homogénéité de ses fonctionnaires et de la nébuleuse de ceux avec qui ils travaillent (consultants internationaux ou locaux, entre autres). Tous partagent le même modèle idéologique, fait d'orthodoxie économique et de culture bureaucratique spécifique, forgé au fil des ans. La prépondérance de la fonction financière tend à renforcer cette cohésion, dont l'impératif de décaissement demeure une valeur sûre. Certes, la Banque n'est pas monolithique, mais le débat y reste très largement encadré. Le mode de recrutement et les mécanismes de promotion cimentent cette unité d'identité.

Finalement, en dépit des évolutions et des réformes, l'action de la Banque, les impulsions au sommet de même que les pratiques à la base et au quotidien sont toutes façonnées par le même paradigme économiciste mainstream, à l'aune duquel sont absorbées les nouvelles thématiques et les nouvelles approches, un temps considérées comme « hors champ ». C'est d'ailleurs au nom des théories du public choice que le Président R. Zoellick justifie l'incursion dans la participation citoyenne et l'appui aux organisations de la société civile. Cette approche mono-disciplinaire risque de continuer à repousser aux calendes grecques l'embauche significative d'autres spécialistes de sciences sociales (sociologues, anthropologues, politistes, etc.) aux côtés du gros bataillon des économistes, pour traiter des questions de développement, par ailleurs reconnues comme multidisciplinaires. L’hégémonie que la Banque mondiale s'est construite en matière de savoir sur le développement est en totale contradiction avec les principes du marché qu'elle préconise. En l'absence de concurrence et de véritable contre-pouvoir, rien finalement n'oblige la Banque, qui jouit d'une position dominante, à revoir les principes prégnants et sous-jacents aux fondements de ses politiques, et à explorer sérieusement des alternatives ${ }^{39}$.

39. Ce constat rejoint celui d’O. Nay soulignant de façon plus générale la nécessité pour les institutions internationales d'être plus ouvertes et d'instaurer une relation de partenariat d'égal à égal avec les autres acteurs du développement pour permettre une véritable réforme des politiques et des 
Paradoxalement, la crise internationale de 2008 constitue une aubaine pour la Banque mondiale (et encore plus pour le FMI qui était moribond) ${ }^{40}$. Elle lui a permis d'augmenter considérablement ses financements, et même de trouver une future excuse au fait que les OMD ne seront pas atteints à l'horizon 2015 : ainsi la Banque évite-t-elle toute critique, au demeurant prévisible, quant à l'efficacité de ses politiques de réduction de la pauvreté.

En même temps, force est de constater qu'en cette période de crise le principal centre d'attention et par là même le principal gagnant est, plus que la Banque mondiale, le FMI. Ce sont en effet les pays développés qui sont les clients du FMI, lequel joue un rôle clé dans la survie du système financier international et de la zone euro ; en revanche, les seuls clients de la Banque mondiale sont les PED et l'importance systémique de l'institution est beaucoup plus réduite. Ce désintérêt relatif (hormis peut-être pour les questions sécuritaires et migratoires) est lourd de conséquences puisqu'il hypothèque toute réforme en profondeur, pourtant nécessaire, de l'architecture économique internationale et plus spécifiquement de la Banque dans un proche avenir. Finalement, ce sont les crises récurrentes du système mondialisation libérale et capitalisme - dont ils sont les fervents promoteurs qui assurent la pérennité institutionnelle de la Banque mondiale et du FMI.

pratiques dans le champ du développement. O. Nay, «Les politiques de développement », dans Olivier Borraz, Virginie Guiraudon (dir.), Les politiques publiques 2. Changer la société, Paris, Presses de Sciences Po, 2010, p. 139-170.

40. En 2008 encore, C. E. Weaver, spécialiste reconnue de l'institution, affirmait: «La Banque mondiale est aujourd'hui en crise et (...) le chemin pour la survie précaire ». C. E. Weaver, Hypocrisy Trap: The World Bank and the Poverty of Reform, op. cit. (nous traduisons). 
Jean-Pierre Cling est professeur d'économie à l'Université Paris Nord (UMR CEPN-CNRS), où il enseigne l'économie internationale et du développement. Il a notamment publié (avec Mireille Razafindrakoto et François Roubaud) Les Nouvelles Stratégies internationales de lutte contre la pauvreté (Paris, Économica, 2003), La Banque mondiale (Paris, La Découverte, 2008) et La Banque mondiale à la recherche de modèles de développement : le cas du Vietnam (Hanoï, Tri Thuc, 2009). Adresse électronique : cling@univ-paris13.fr

Mireille Razafindrakoto est économiste, directrice de recherche à l'IRD et membre de I'UMR DIAL. Ses thèmes de recherche sont la gouvernance et les institutions dans les PED, l'impact des politiques publiques sur les conditions de vie des ménages, ainsi que le marché du travail et les multiples dimensions de la pauvreté. Adresse électronique : razafindrakoto@dial.prd.fr

François Roubaud est économiste, directeur de recherche à l'IRD et membre de l'UMR DIAL. Outre ses travaux dans le champ de l'économie politique, ses domaines de spécialité sont le marché du travail et l'économie informelle, l'évaluation des politiques de développement et les questions de gouvernance. Adresse électronique : roubaud@dial.prd.fr 